\title{
In-plane vibration of rotating rings using a high order the- ory
}

\author{
Tao $\mathrm{Lu}^{1, *}$, Apostolos Tsouvalas ${ }^{1}$, and Andrei Metrikine ${ }^{1}$ \\ ${ }^{1}$ Faculty of Civil Engineering and Geosciences, Delft University of Technology, Stevinweg 1, 2628CN \\ Delft, The Netherlands
}

\begin{abstract}
In-plane dynamics of rotating rings on elastic foundation is a topic of continuous research, especially in the field of tire dynamics. When the inner surface of a ring is connected to a stiff foundation, the through-thickness variation of radial and shear stress needs to be accounted for. This effect is often overlooked in the ring models proposed in the literature. In this paper, a new high order theory is developed for the in-plane vibration of rotating rings whose inner surface is connected to an immovable hub by distributed springs while the outer surface is stress-free. The high-order terms are chosen such that the boundary conditions at the inner and outer surfaces are satisfied at all times. Instability, which is usually overlooked in the literature, is predicted using the present model. Resonant speeds are investigated, at which modes appear as a stationary displacement pattern to a space-fixed observer. The exact satisfaction of boundary conditions at the inner and outer ring surfaces together with the through-thickness variation of the radial and shear stresses are shown to be of significant importance when the ring rotates at high speeds or is supported by relatively stiff foundation.
\end{abstract}

\section{Derivation of governing equations}

As is shown in Fig. 1, the model consists of a flexible ring of rectangular cross-section and distributed radial and circumferential springs (designated as $k_{r}$ and $k_{c}$ ) that connect the inner surface of the ring to an immovable axis. The ring rotates at an angular speed $\Omega$. A spacefixed coordinate system $(r, \theta)$ is adopted. It is assumed that the mean radius of the ring is $R$. To simplify mathematical expressions, an auxiliary coordinate $z$ is introduced as $z=r-R$, in which $r$ defines the radial coordinate, i.e. the ring occupies the space $R-h / 2 \leq r \leq R+h / 2$, in which $h$ denotes the thickness of the ring. The radial and circumferential displacements of the ring are designated by $w(z, \theta, t)$ and $u(z, \theta, t)$, respectively. Furthermore, $\rho$ is the mass density of the ring, $E$ is the Young's modulus, $A$ is the cross-sectional area and $I$ is the cross-sectional moment of inertia, and $b$ is the out-of-plane width of the ring.

The displacements $w(z, \theta, t)$ and $u(z, \theta, t)$ of a differential element of the ring are defined by

$$
\begin{aligned}
& w(z, \theta, t)=w_{0}(\theta, t)+z w_{1}(\theta, t)+z^{2} w_{2}(\theta, t) \\
& u(z, \theta, t)=u_{0}(\theta, t)+z \phi_{1}(\theta, t)+z^{2} \phi_{2}(\theta, t)+z^{3} \phi_{3}(\theta, t)
\end{aligned}
$$

*e-mail: T.Lu-2@tudelft.nl 


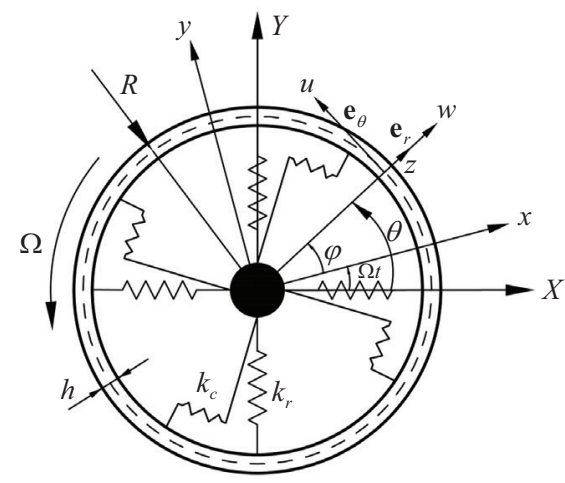

Figure 1. A rotating ring on an elastic foundation

in which $w_{0}(\theta, t)$ and $u_{0}(\theta, t)$ are the radial and circumferential displacements of the middle surface, respectively. The terms $w_{1}(\theta, t), w_{2}(\theta, t), \phi_{2}(\theta, t)$ and $\phi_{3}(\theta, t)$ are high order corrections of the radial and circumferential displacements which enable one to take into consideration the through-thickness variation of the radial and shear stresses.

According to [1], the nonlinear strain-displacement relation for the circumferential strain $\varepsilon_{\theta}$, the radial strain $\varepsilon_{r}$ and the shear strain $\gamma_{\theta r}$ of a differential element in the ring are given by

$$
\varepsilon_{\theta}=\varepsilon_{0}+\frac{1}{2}(\beta)^{2}, \varepsilon_{r}=w_{, r}+\frac{1}{2}\left(u_{, r}\right)^{2}, \gamma_{\theta r}=\left(1-w_{, r}\right) u_{, r}-\beta \eta
$$

with:

$$
\begin{aligned}
& \varepsilon_{0}=\frac{u^{\prime}}{r}+\frac{w}{r}, \beta=\frac{u}{r}-\frac{w^{\prime}}{r}, \eta=1-\varepsilon_{0}, \\
& u_{, r}=\frac{\partial u}{\partial z}=\phi_{1}+2 z \phi_{2}+3 z^{2} \phi_{3}, w_{, r}=\frac{\partial w}{\partial z}=w_{1}+2 z w_{2} .
\end{aligned}
$$

Hereafter, the prime stands for the partial derivative with respect to $\theta$. Please note that all strain components are functions of $(z, \theta, t)$, e.g. $\varepsilon_{\theta} \rightarrow \varepsilon_{\theta}(z, \theta, t)$. The in-plane motions of a ring can either be considered as a plane strain or plane stress problem depending on the ratio $b / h$. When $b / h>>1$, the problem is essentially of the plane strain type. Without loss of generality, the isotropic linear elastic stress-strain relations are [2]:

$$
\left\{\begin{array}{l}
\sigma_{r} \\
\sigma_{\theta} \\
\tau_{r \theta}
\end{array}\right\}=\left[\begin{array}{ccc}
2 \mu+\bar{\lambda} & \bar{\lambda} & 0 \\
\bar{\lambda} & 2 \mu+\bar{\lambda} & 0 \\
0 & 0 & \mu
\end{array}\right]\left\{\begin{array}{c}
\varepsilon_{r} \\
\varepsilon_{\theta} \\
\gamma_{r \theta}
\end{array}\right\}
$$

in which $\bar{\lambda}=\lambda$ for the plane strain configuration; and $\bar{\lambda}=2 \mu \lambda /(2 \mu+\lambda)=E v /\left(1-v^{2}\right)$ for the plane stress case. $\lambda$ and $\mu$ are the Lamé constants of the material and $v$ is the Poisson's ratio.

The inner surface of the ring is connected by means of distributed radial and circumferential springs to an immovable axis. The radial and shear stresses should be zero at the outer surface,

$$
\begin{aligned}
& \left.\sigma_{r}\right|_{h / 2}=2 \mu\left(\left.\varepsilon_{r}\right|_{h / 2}\right)+\bar{\lambda}\left(\left.\varepsilon_{r}\right|_{h / 2}+\left.\varepsilon_{\theta}\right|_{h / 2}\right)=0, \\
& \left.\tau_{\theta r}\right|_{h / 2}=\left.\mu \gamma_{\theta r}\right|_{h / 2}=0 .
\end{aligned}
$$


The inner surface of the ring is connected to foundation, thus

$$
\begin{aligned}
& \left.\sigma_{r}\right|_{-h / 2}=2 \mu\left(\left.\varepsilon_{r}\right|_{-h / 2}\right)+\bar{\lambda}\left(\left.\varepsilon_{r}\right|_{-h / 2}+\left.\varepsilon_{\theta}\right|_{-h / 2}\right)=k_{r}\left(w_{0}-w_{1} h / 2+w_{2} h^{2} / 4\right), \\
& \left.\tau_{\theta r}\right|_{-h / 2}=\left.\mu \gamma_{\theta r}\right|_{-h / 2}=\left.k_{c} u\right|_{-h / 2}=k_{c}\left(u_{0}-\phi_{1} h / 2+h^{2} \phi_{2} / 4-h^{3} \phi_{3} / 8\right) .
\end{aligned}
$$

It is assumed that $w_{0}, u_{0}$ and $\phi_{1}$ are independent variables, whereas $w_{1}, w_{2}, \phi_{2}$ and $\phi_{3}$ can be expressed as functions of $w_{0}, u_{0}$ and $\phi_{1}$ by satisfying Eqs. (5-6) above:

$$
\begin{aligned}
& w_{1}(\theta, t)=w_{1}\left(w_{0}, u_{0}, \phi_{1}\right), w_{2}(\theta, t)=w_{2}\left(w_{0}, u_{0}, \phi_{1}\right), \\
& \phi_{2}(\theta, t)=\phi_{2}\left(w_{0}, u_{0}, \phi_{1}\right), \phi_{3}(\theta, t)=\phi_{3}\left(w_{0}, u_{0}, \phi_{1}\right) .
\end{aligned}
$$

The velocity vector in the space-fixed frame reads

$$
\dot{\mathbf{r}}=\left(\dot{w}+\left(w^{\prime}-u\right) \Omega\right) \mathbf{i}+\left(\dot{u}+\left(r+w+u^{\prime}\right) \Omega\right) \mathbf{j}=v_{1} \mathbf{i}+v_{2} \mathbf{j} .
$$

The vectors $\mathbf{i}$ and $\mathbf{j}$ are unit vectors in the radial and circumferential directions, respectively. The overdot denotes a partial derivative with respect to time.

Application of Hamilton's principle, i.e.

$$
\begin{aligned}
& \int_{t_{1}}^{t_{2}} \int_{\frac{-h}{2}}^{\frac{h}{2}} \int_{0}^{2 \pi}\left(\sigma_{\theta} \delta \varepsilon_{\theta}+\sigma_{r} \delta \varepsilon_{r}+\tau_{\theta r} \delta \gamma_{\theta r}\right) r \mathrm{~d} \theta \mathrm{d} z \mathrm{~d} t-\frac{\rho}{2} \int_{t_{1}}^{t_{2}} \int_{\frac{-h}{2}}^{\frac{h}{2}} \int_{0}^{2 \pi} \delta(\dot{\mathbf{r}} \cdot \dot{\mathbf{r}}) r \mathrm{~d} \theta \mathrm{d} z \mathrm{~d} t \\
& +\int_{t_{1}}^{t_{2}} \int_{0}^{2 \pi}\left(\left.k_{r} w\right|_{-h / 2} \delta\left(\left.w\right|_{-h / 2}\right)+\left.k_{c} u\right|_{-h / 2} \delta\left(\left.u\right|_{-h / 2}\right)\right)(R-h / 2) \mathrm{d} \theta \mathrm{d} t=0
\end{aligned}
$$

yields three nonlinear dynamic equations by setting the coefficients of $\delta w_{0}, \delta u_{0}$ and $\delta \phi_{1}$ equal to zero:

$$
\begin{aligned}
& \int_{\frac{-h}{2}}^{\frac{h}{2}}\left(A_{1} \mathrm{I}_{1}\right) \mathrm{d} z+\int_{\frac{-h}{2}}^{\frac{h}{2}}\left(A_{2} \mathrm{I}_{2}\right) \mathrm{d} z+\int_{\frac{-h}{2}}^{\frac{h}{2}}\left(A_{3} \mathrm{I}_{3}\right) \mathrm{d} z+\int_{\frac{-h}{2}}^{\frac{h}{2}}\left(A_{4} \mathrm{I}_{4}\right) \mathrm{d} z \\
& +\rho \int_{\frac{-h}{2}}^{\frac{h}{2}}\left(\left(\dot{v}_{1}+\Omega v_{1}^{\prime}-\Omega v_{2}\right) A_{1}+\left(\dot{v}_{2}+\Omega v_{2}^{\prime}+\Omega v_{1}\right) A_{2}\right) r \mathrm{~d} z \\
& +\left(\left.k_{r} c_{1} w\right|_{-h / 2}+\left.k_{c} c_{4} u\right|_{-h / 2}\right)(R-h / 2)=0, \\
& \int_{\frac{-h}{2}}^{\frac{h}{2}}\left(B_{1} \mathrm{I}_{1}\right) \mathrm{d} z+\int_{\frac{-h}{2}}^{\frac{h}{2}}\left(B_{2} \mathrm{I}_{2}\right) \mathrm{d} z+\int_{\frac{-h}{2}}^{\frac{h}{2}}\left(B_{3} \mathrm{I}_{3}\right) \mathrm{d} z+\int_{\frac{-h}{2}}^{\frac{h}{2}}\left(B_{4} \mathrm{I}_{4}\right) \mathrm{d} z \\
& +\rho \int_{\frac{-h}{2}}^{\frac{h}{2}}\left(\left(\dot{v}_{1}+\Omega v_{1}^{\prime}-\Omega v_{2}\right) B_{1}+\left(\dot{v}_{2}+\Omega v_{2}^{\prime}+\Omega v_{1}\right) B_{2}\right) r \mathrm{~d} z \\
& +\left(\left.k_{r} c_{2} w\right|_{-h / 2}+\left.k_{c} c_{5} u\right|_{-h / 2}\right)(R-h / 2)=0, \\
& \int_{\frac{-h}{2}}^{\frac{h}{2}}\left(C_{1} \mathrm{I}_{1}\right) \mathrm{d} z+\int_{\frac{-h}{2}}^{\frac{h}{2}}\left(C_{2} \mathrm{I}_{2}\right) \mathrm{d} z+\int_{\frac{-h}{2}}^{\frac{h}{2}}\left(C_{3} \mathrm{I}_{3}\right) \mathrm{d} z+\int_{\frac{-h}{2}}^{\frac{h}{2}}\left(C_{4} \mathrm{I}_{4}\right) \mathrm{d} z \\
& +\rho \int_{\frac{-h}{2}}^{\frac{h}{2}}\left(\left(\dot{v}_{1}+\Omega v_{1}^{\prime}-\Omega v_{2}\right) C_{1}+\left(\dot{v}_{2}+\Omega v_{2}^{\prime}+\Omega v_{1}\right) C_{2}\right) r \mathrm{~d} z \\
& +\left(\left.k_{r} c_{3} w\right|_{-h / 2}+\left.k_{c} c_{6} u\right|_{-h / 2}\right)(R-h / 2)=0,
\end{aligned}
$$

where

$$
\begin{aligned}
& \mathrm{I}_{1}=\sigma_{\theta}+\left(\sigma_{\theta} \beta\right)^{\prime}-\left(\tau_{\theta r} \eta\right)^{\prime}+\tau_{\theta r} \beta, \quad \mathrm{I}_{2}=-\left(\sigma_{\theta}\right)^{\prime}+\sigma_{\theta} \beta-\tau_{\theta r} \eta-\left(\tau_{\theta r} \beta\right)^{\prime} \\
& \mathrm{I}_{3}=\left(\sigma_{r}-\tau_{\theta r} u_{, r}\right) r, \quad \mathrm{I}_{4}=\left(\sigma_{r} u_{, r}+\tau_{\theta r}\left(1-w_{, r}\right)\right) r
\end{aligned}
$$


The coefficients $A_{1}-A_{4}, B_{1}-B_{4}, C_{1}-C_{4}$ and $c_{1}-c_{6}$ can be obtained by combining Eqs. (1) and (7).

The nonlinear governing equations are then linearised around the static equilibrium resulting from rotation. The static equilibrium is solved from the nonlinear governing equations the same way as Ref. [3]. To obtain to characteristic equation from the linearised governing equations, it is assumed that the dynamic displacements are

$$
w_{0}(\theta, t)=R W \mathrm{e}^{\mathrm{i} n \theta+\mathrm{i} \omega t}, u_{0}(\theta, t)=R U \mathrm{e}^{\mathrm{i} n \theta+\mathrm{i} \omega t}, \phi_{1}(\theta, t)=\Phi \mathrm{e}^{\mathrm{i} n \theta+\mathrm{i} \omega t}
$$

in which $\omega$ is the natural frequency in space-fixed reference system, $n$ is the circumferential mode number and $\mathrm{i}=\sqrt{-1}$. Substituting Eq. (14) into the linearised governing equations yields the characteristic equation. For convenience, the following dimensionless parameters are introduced

$$
k=\sqrt{E I /(E A)}, \bar{k}=k / R, \bar{\gamma}=n \bar{k}, \bar{\omega}=\omega k / c_{0}, \bar{v}=R \Omega / c_{0},\left(\bar{k}_{r}, \bar{k}_{c}\right)=\left(k_{r}, k_{c}\right) k^{2} /(E h)
$$

where $c_{0}=\sqrt{E / \rho}$ is the longitudinal wave speed of a rod, $I=b h^{3} / 12$ is the cross section area moment of inertia and $\bar{k}$ is the non-dimensional radius of gyration. The dimensionless characteristic equation is then expressed by

$$
f(\bar{\omega}, \bar{\gamma}, \bar{v})=0 .
$$

\section{Comparison with linear elasticity for the stationary ring case}

To illustrate the significance of satisfaction of boundary conditions, the proposed model is compared with the Timoshenko-type model [4] and elasticity theory for the stationary ring case $(\Omega=0)$. Plane strain is assumed since we consider a ring with $b>>h$.
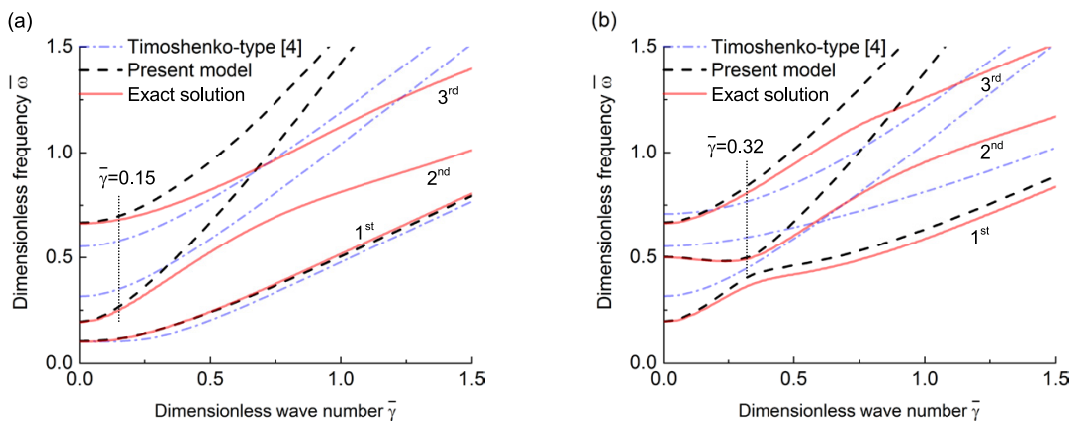

Figure 2. Frequency spectrum (plane strain) for $h / R=0.1, \bar{k}_{c}=0.1$ and $\Omega=0$ : (a) $\bar{k}_{r}=0.01$; (b) $\bar{k}_{r}=0.5$.

The frequency spectra are plotted in Fig. 2 for two sets of foundation stiffness and for $v=0.4$. The first three branches of the frequency spectra calculated by the elasticity theory are plotted to compare with the Timoshenko-type theory and the high-order theory proposed here. It can be concluded that the applicability range of the new model is much wider than the Timoshenko-type model [4]. The Timoshenko model is not able to capture the dispersion curves when the foundation stiffness increases as shown in Fig. 2(b) even for the lowest branch of the frequency spectra. In contrast, the current model can accurately predict the 
lowest branch in the whole range of wavenumbers. For the higher branches, the high-order theory is valid till $\bar{\gamma} \approx 0.15$ in Fig. 2(a) and $\bar{\gamma} \approx 0.32$ in Fig. 2(b). To conclude, the high-order theory which considers the boundary conditions at the surfaces and the thoughthickness variations of stresses is shown to be superior to Timoshenko-type ring models.

\section{Critical speeds of rotating rings}

\subsection{Resonant speeds}

Resonant speeds of a rotating ring are defined as the speeds at which resonances of the ring subjected to a stationary load occur. They are the speeds which satisfy the condition $\bar{\omega}=0$ $[3,5]$, namely the natural frequency in the space-fixed coordinate becomes zero at these speeds. By substituting $\bar{\omega}=0$ into the characteristic equation (16), one can solve for resonant speeds for each circumferential wavenumber.
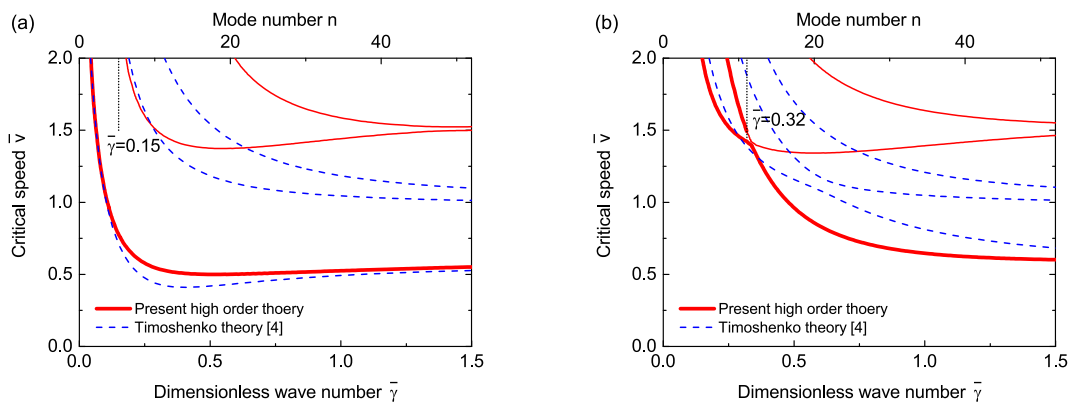

Figure 3. Resonant speeds for $h / R=0.1, \bar{k}_{c}=0.1$ : (a) $\bar{k}_{r}=0.01$; (b) $\bar{k}_{r}=0.5$.

Figure 3 shows the resonant speeds for $\bar{k}_{r}=0.01$ and $\bar{k}_{r}=0.5$ whick $\bar{k}_{c}=0.1$. Three curves which correspond to the three dispersion curves shown in Fig. 2 exist. The vertical dotted line in Fig. 3(a)(b) corresponds to wavenumber $\bar{\gamma}=0.15$ and $\bar{\gamma}=0.32$ which are obtained from Fig. 2 marking the applicable range for the higher branches. That is to say, the thicker solid lines are of interest from the predictions of the present high order theory. The lower abscissa in each plot is the dimensionless wavenumber, whereas the upper abscissa is the corresponding discrete circumferential mode number. In Fig. 3(a), the two models predict qualitatively similar resonant speeds. The resonant speeds of the lowest branch approach the Rayleigh wave speed at large wavenumbers. To conclude, if the foundation is relatively soft, the first order Timoshenko correction gives accurate results on the prediction of resonat speeds. However, it underestimates the minimum resonant speed. In Fig. 3(b), we note that the influence of boundary conditions increases. The quantitatively different resonant speeds predicted in Fig. 3(b) show the significance of boundary conditions for the case of stiff foundation.

\subsection{Critical speeds associated with instability}

It has been shown in [3] that instability may occur for certain combination of parameters. Divergence instability of the $n=0$ rotational mode always occurs at lower rotational speeds compared to flutter of higher modes.

In a previous study [3], it is concluded that the stiffness of the circumferential springs is a key factor for determining the critical speed corresponding to the onset of instability. Here 

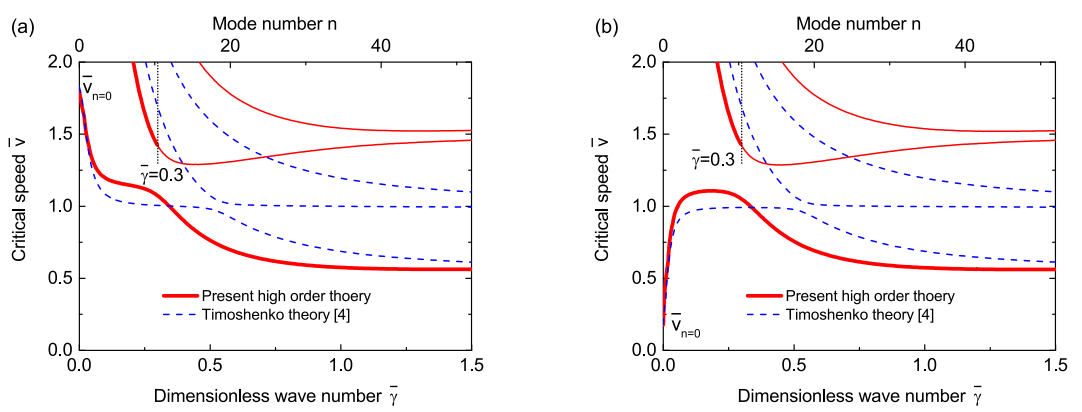

Figure 4. Critical speeds for $h / R=0.1, \bar{k}_{r}=0.28$ : (a) $\bar{k}_{c}=10^{-2} \bar{k}_{r}$; (b) $\bar{k}_{c}=10^{-4} \bar{k}_{r}$.

we choose two sets of parameters, among them $\bar{k}_{r}=0.28$ corresponds to the stiffness of radial springs $\bar{K}_{r}=4 \times 10^{5}$ used in [3] and $\bar{k}_{c}$ is different. In each figure, $\bar{v}_{n=0}$ denotes the critical speed associated with the onset of instability of mode $n=0$. Besides the resonant speeds for higher modes, the focus is placed to the critical speeds for instability of $n=0$ mode. Two conclusions can be drawn from Fig. 4. First, the speeds that correspond to the divergence instability are quite close to each other, regardless of the models employed. The proposed model predicts slightly smaller critical speed of $n=0$ which means that the consideration of boundary conditions lowers the critical speeds for $n=0$. Second, the critical speeds of $n=0$ can be smaller than the resonant speeds of higher modes, implying that the ring may become unstable before resonances could occur.

\section{Conclusions}

In this paper, the in-plane vibration of a rotating ring on a relatively stiff elastic foundation is considered. A new high order model is developed which includes the shear deformation, the rotatory inertia and the through-thickness variation of the radial and shear stresses. The present model can treat both plane strain and plane stress problems, as well as stationary rings by setting the rotational speed of the ring to zero. The boundary conditions at the inner and outer surfaces of the ring are satisfied in an exact manner. Two types of critical speeds are discussed. The first one corresponds to resonances of a rotating ring subjected to a stationary load. The second one is responsible for divergence instability of the free vibration of a rotating ring. By analysing the critical speeds using different models, it is shown that the shear deformation and rotatory inertia are important. The influence of radial and shear stress boundary conditions becomes significant when the foundation is relatively stiff, especially for waves of long wavelength.

\section{References}

[1] M. Stein, AIAA J. 24, 1537 (1986)

[2] H. Matsunaga, Acta Mech. 124, 47 (1997)

[3] T. Lu, A. Tsouvalas, A. Metrikine, J. Sound Vib. 402, 203 (2017)

[4] H. Ding, M. Zhu, Z. Zhang, Y.-W. Zhang, L.-Q. Chen, Int. J. Appl. Mechanics 09, 1750051 (2017)

[5] W. Soedel, Vibrations of Shells and Plates (CRC Press, 2004) 